\title{
Manifolds with non-positive curvature
}

\author{
K. BURNS AND A. KATOK \\ In collaboration with W. Ballman, M. Brin, P. Eberlein and R. Osserman \\ University of Maryland, College Park, MD 20742, USA: California Institute of \\ Technology, Pasadena, CA 91125, USA
}

(Received 18 July 1984 and revised 14 February 1985)

\begin{abstract}
This is an extended report on a problem session about geodesic flows held on May 30, 1984 at MSRI, Berkeley. The list of six authors reflects the principal participants in the discussion. Contributions of other participants are mentioned in the text. The session was chaired by A. Katok; this report was prepared by K. Burns in collaboration with $\mathrm{A}$. Katok.
\end{abstract}

\section{0 . Notation and definitions}

$M$ is a connected $C^{\infty}$ Riemannian manifold with sectional curvature $K_{M}$ and curvature tensor $R$. Unless otherwise stated, it is assumed that $K_{M} \leq 0$. Also geodesics have unit speed.

$I(M)$ is the isometry group of $M$.

$\tilde{M}$ is the universal cover of $M$.

$\tilde{M}(\infty)$ is the sphere of points at infinity for $\tilde{M}$.

$S M$ is the unit tangent bundle of $M$.

$g^{t}$ is the geodesic flow on $S M$ or $S \tilde{M}$.

$\mu$ is the Liouville measure on $S M$, normalized so that $\mu(S M)=1$.

$h$ is the topological entropy of $g^{t}$.

$h_{\nu}$ is the measure theoretic entropy of $g^{t}$ with respect to an invariant probability

$\nu$. The metric entropy $h_{\mu}$ is especially important.

$\gamma_{v}$ is the geodesic defined by a vector $v \in S M$ (or $S \tilde{M}$ ).

$v$ is a periodic vector if $\gamma_{v}$ is closed.

The rank of $v \in S M$ is the dimension of the space of parallel Jacobi fields along $\gamma_{v}$. The rank of $M$ is the minimum rank of a vector in $S M$. This agrees with the usual definition when $M$ is locally symmetric. If $\operatorname{rank}(v)=\operatorname{rank}(M)$, then $v$ and $\gamma_{v}$ are called regular. A $k$-flat in $M$ is a $k$-dimensional Euclidean space totally geodesically and isometrically immersed in $M$. A $k$-flat $F$ is regular if $k=\operatorname{rank}(M)$ and $F$ contains a regular geodesic.

\section{Some recent results}

Suppose $M$ has finite volume, rank $k$, and curvature bounded below.

(1.1) The set of regular vectors is open and dense in $S M$, and every regular vector is tangent to a unique $k$-flat. This holds even without the curvature bound. [5]. 
(1.2) Periodic regular vectors are dense in $S M$, and if $M$ is compact the $k$-flat to which such a vector is tangent is compact. [6].

(1.3) Dichotomy. If $M$ is compact, the geodesic flow $g^{\prime}$ is ergodic if and only if $M$ has rank 1. [11], [4], [5].

(1.4) Classification in higher rank. If $k \geq 2$, the de Rham factors [28] of $\tilde{M}$ are

(i) Euclidean;

(ii) of rank 1 , or

(iii) symmetric spaces.

(1.4') If in addition $\tilde{M}$ has no Euclidean factor, $M$ has a finite cover $M^{\prime}$ which is a product of locally symmetric spaces and rank 1 manifolds.

(1.4) and (1.4') have been proved by Ballmann [47]. Spatzier and Burns also have a proof for compact $M$; their manuscript is in preparation.

(1.5) Rigidity for locally symmetric spaces. Suppose $M$ is compact and $\pi_{1}(M) \cong$ $\pi_{1}\left(M^{*}\right)$, where $M^{*}$ is a compact irreducible locally symmetric manifold of rank $\geq 2$. Then $M$ and $M^{*}$ are isometric provided the metric of one of them is multiplied by a suitable constant.

This is due to Eberlein and Gromov. See [15] and [38].

(1.6) [36] is a comprehensive survey of results up to 1981. Also [40] surveys the results in [5] and [6].

\section{2: Possible generalizations}

(2.1) (Eberlein). Let $\Gamma$ be a (not necessarily discrete) subgroup of $I(\tilde{M})$.

(2.1.1) Definition. The non-wandering set of $g^{\prime} \bmod \Gamma$ is $\Omega(\Gamma)=\{v \in S \tilde{M}$ : if $O \subseteq S \tilde{M}$ is any neighbourhood of $v$, then there exist sequences $\left\{\varphi_{n}\right\} \subseteq \Gamma$ and $t_{n} \rightarrow+\infty$ such that $\left(d \varphi_{n} \circ g^{t_{n}}\right)(O) \cap O \neq \varnothing$ for all $\left.n\right\}$.

Consider groups $\Gamma$ with $\Omega(\Gamma)=S \tilde{M}$. This property holds when $\tilde{M} / \Gamma$ is a smooth manifold with finite volume, and also more generally (see remark (2) on p. 163 of [13]). It is equivalent to the duality condition of [12]. Note that:

(i) If $\Gamma$ preserves any Riemannian splitting $\tilde{M}=\tilde{M}_{1} \times \cdots \times \tilde{M}_{k}$, then $\Omega\left(p_{i}(\Gamma)\right)=$ $S \tilde{M}_{i}$, for $1 \leq i \leq k$, where $p_{i}: \Gamma \rightarrow I\left(\tilde{M}_{i}\right)$ is the projection homomorphism.

(ii) If $\Omega(\Gamma)=S \tilde{M}$, then $\Omega\left(\Gamma^{*}\right)=S \tilde{M}$ for any finite index subgroup $\Gamma^{*} \subseteq \Gamma$.

(iii) If $\Omega(I(\tilde{M}))=S \tilde{M}$, then $\tilde{M}=\mathbb{R}^{P} \times$ symmetry space $\times B$, where $I(B)$ is discrete and $\Omega(I(B))=S B$.

Can one give a better description of $\Gamma$ if $\Omega(\Gamma)=S \tilde{M}$ ? By (i) it suffices to consider $\tilde{M}$ Euclidean or irreducible (not a product). It follows from (iii) that if $\tilde{M}$ is irreducible, then either $\Gamma$ is discrete or $\tilde{M}$ is symmetric and $(\bar{\Gamma})_{0}=I_{0}(\tilde{M})$.

(2.1.2) Conjecture (Eberlein). If $\tilde{M}$ is irreducible, $\operatorname{rank}(\tilde{M}) \geq 2$ and $\Omega(I(\tilde{M}))=S \tilde{M}$, then $\tilde{M}$ is a symmetric space. This would generalize (1.4). In particular it would mean that (1.4) holds without the lower bound on $K$.

(2.1.3) Conjecture (Eberlein). If $\tilde{M}$ is symmetric of rank $\geq 2$ and $\Omega(\Gamma)=S \tilde{M}$ with $\Gamma \subseteq I(\tilde{M})$ discrete, then $\Gamma$ is a lattice (i.e. $\Gamma$ has a torsion free subgroup $\Gamma_{0}$ of finite index such that $\tilde{M} / \Gamma_{0}$ has finite volume). 
(2.2) Problem. Is $g^{t}$ ergodic if $M$ has rank 1 , finite volume and bounded curvature? If the lower bound on the curvature is dropped?

(2.3) It should be easy to extend (1.4) to manifolds with no focal points.

(2.4) (Spatzier). It follows from (1.4) that there is an $\mathbb{R}^{k}$ action on $S M$, where $k=\operatorname{rank}(M)$. Can (1.4) be generalized to a rigidity result for (non-uniformly) hyperbolic $\mathbb{R}^{k}$ actions?

(2.5) (Hurder). Let $M$ be a $C^{\infty}$ manifold and $m$ a finite measure on $M$. Let $\mathscr{F}$ be a measurable foliation of $M$ with $C^{\infty}$ leaves. Let $g$ be a Riemannian metric on the leaves of $\mathscr{F}$ which is smooth on each leaf and measurable on $M$. Denote the Riemannian volume on leaves by $\lambda_{g}$ and define the transverse measure $m_{T}$ by $m=m_{T} \times \lambda_{\mathrm{g}}$.

\section{(2.5.1) Conjecture. Suppose}

(i) $g$ has non-positive curvature and every leaf is irreducible and has rank $k \geq 2$.

(ii) $\mathscr{F}$ is ergodic with respect to $m_{T}$.

Then there is a symmetric space $S$ of rank $k$ such that almost every leaf of $\mathscr{F}$ is modelled on $S$.

(2.5.2) This reduces to (1.4) when $\mathscr{F}$ has just one leaf. The point is to extend (1.4) to manifolds of infinite volume which are leaves in foliations with finite total volume.

(2.5.3) For a related question, see $[23, \S 3]$.

\section{The length spectrum}

It is well known that any Riemannian metric $\sigma$ of negative curvature on a compact manifold has a unique closed geodesic in every free homotopy class $\gamma$. Let $l_{\sigma}(\gamma)$ be the length of this geodesic. Equivalently $l_{\sigma}(\gamma)$ is the minimum length of a smooth loop representing $\gamma$. We call the function $l_{\sigma}$ the length spectrum of $\sigma$.

(3.1) Conjecture. Let $\sigma$ and $\sigma^{*}$ be two metrics of negative curvature on the same compact manifold $M$. If $\sigma$ and $\sigma^{*}$ have the same length spectrum, there is a diffeomorphism of $M$ which carries $\sigma$ to $\sigma^{*}$.

Note that the set of lengths of closed geodesics does not determine the isometry type of $M$, even if $M$ is a surface of constant negative curvature; see [44].

We now discuss some partial results in the direction of the conjecture.

(3.2) Fricke and Klein [18] showed that the assertion of the conjecture holds if $M$ is a surface and $\sigma$ and $\sigma^{*}$ both have constant negative curvature. For an outline of the argument, see [30].

(3.3) Guillemin and Kazhdan [22] have shown that for a surface of genus $\geq 2$ the assertion of the conjecture holds provided the two metrics can be joined by a smooth path of metrics along which the length spectrum is constant. They also have a similar result in higher dimensions with some pinching conditions on the metric.

(3.4) Katok has shown that if the two metrics are conformally equivalent and have the same length spectrum, then the assertion of the conjecture holds. This can be extracted from the results of [25]. Since for surfaces the space of conformal 
equivalence classes of metrics (Teichmüller space) is finite dimensional, this strongly suggests that the conjecture is true for surfaces.

(3.5) (cf. 10.3) Two metrics on a surface which have the same length spectrum have their geodesic flows conjugate by a $C^{1}$ diffeomorphism of the unit tangent bundle. The proof relies on the fact that the set of all metrics of negative curvature is connected, and first establishes the existence of a Hölder conjugacy. Then it follows from [17] that the conjugacy is actually $C^{1}$. The connectedness of the set of negatively curved metrics is not known at present in higher dimensions; see (7.1) below.

(3.6) We can see from (3.2) and (3.5) together with [25] that the conjecture holds if the manifold is a surface and one of the metrics has constant negative curvature. For the length spectrum determines the topological entropy $h$, and by (3.5) it also determines the metric type of the geodesic flow with respect to the Liouville measure $\mu$, and hence $h_{\mu}$. By [25] $h=h_{\mu}$ if and only if the metric on the surface has constant negative curvature. Thus both metrics have constant curvature and (3.2) applies.

(3.7) Conjecture (3.1) can be extended to the case where $M$ has rank 1 . It is no longer true that a free homotopy class $\gamma$ must contain a unique closed geodesic, but any two closed geodesics in $\gamma$ will have the same length.

(3.8) The length spectrum is intimately connected with the spectrum of the Laplacian. Results about the latter are surveyed in [7], [41].

\section{Statistics of closed geodesics in rank 1}

The hope here is to extend the results for negative curvature to the rank 1 case.

(4.1) Definitions. For $T \geq 0$, let $P(T)$ be the number of free homotopy classes which contain a closed geodesic of length $\leq T$. In the negative curvature case, $P(T)=$ $\# \mathscr{P}(T)$, where $\mathscr{P}(T)$ is the set of closed orbits of $g^{t}$ with period $\leq T$ (we allow multiply-traversed orbits). In the rank 1 case, let $\mathscr{P}_{\text {reg }}(T)$ be the set of orbits from $\mathscr{P}(T)$ which lie inside the set of regular vectors.

(4.2) For a compact manifold with negative curvature, the following results are known.

(4.2.1) $P(T) \sim(h T)^{-1} e^{h T}$ as $T \rightarrow \infty ;(a(T) \sim b(T)$ means $a(T) / b(T) \rightarrow 1)$. See [33], [43], [35].

(4.2.2) The geodesic flow $g^{\prime}$ has a unique invariant probability measure $\mu_{\max }$ such that $h_{\mu_{\max }}=h$. See [8] for existence, [9] for uniqueness.

(4.23) $\mu_{\max }=\lim _{t \rightarrow \infty} L(T)^{-1} \sum_{\sigma \in \mathscr{P}(T)}$ length $(\sigma) \delta_{\sigma}$,

where $L(T)=\sum_{\sigma \in \mathscr{P}(T)}$ length $(\sigma)$ and $\delta_{\sigma}$ is the invariant probability concentrated on the closed orbit of $g^{t}$ corresponding to $\sigma$. See [8].

(4.3) Now consider a compact manifold $M$ with rank 1.

(4.3.1) Knieper [27] has proved that

$$
h=\lim _{T \rightarrow \infty} T^{-1} \log P(T)=\lim _{T \rightarrow \infty} T^{-1} \log \# \mathscr{P}_{\mathrm{reg}}(T)
$$

He uses Manning's result [31] that $h$ is the exponential growth rate of volume of a ball in $\tilde{M}$. 
(4.3.2) Conjecture (Katok). (i) $g^{\prime}$ has a unique measure of maximal entropy, $\mu_{\max }$.

(ii) $\mu_{\max }=\lim _{T \rightarrow \infty} L(T)^{-1} \sum_{\sigma \in \mathscr{P}_{\mathrm{reg}}(T)}$ length $(\sigma) \delta_{\sigma}$, where $\delta_{\sigma}$ is as in (4.2.3) and now $L(T)=\sum_{\sigma \in \mathscr{P}_{\mathrm{reg}}(T)}$ length $(\sigma)$.

(iii) The restriction of $g^{t}$ to the non-regular vectors has entropy $<h$.

\section{Equality of the topological and metric entropies}

In this section $M$ is compact and has negative curvature.

(5.1) Conjecture [25, p. 347]. $h=h_{\mu}$ if and only if $M$ is locally symmetric.

(5.2) There are some partial results.

(5.2.1) $h=h_{\mu}$ when $M$ is locally symmetric [25, p. 346].

(5.2.2) The conjecture is true when $M$ is a surface. This follows from results in [25].

(5.2.3) If the metric on $M$ is conformally equivalent to a locally symmetric metric and $h_{\mu}=h$, then $M$ actually is locally symmetric. See [25].

(5.3) (Katok). One approach is to recall [39] that two functions $\phi$ and $\psi$ have the same equilibrium state with respect to $g^{t}$ if and only if $\phi-\psi$ is cohomologous to the constant function Pressure $(\phi)$-Pressure $(\psi)$. It is known [9] that $\mu$ is the equilibrium state of $\phi^{(u)}(v)=-d /\left.d t \log \lambda_{t}^{u}(v)\right|_{t=0}$, where $\lambda_{t}^{u}(v)$ is the Jacobian of the restriction of $d g^{t}$ to the expanding subspace in $T_{v} S M$. The pressure of $\phi^{(u)}$ is 0 . Also $\mu_{\max }$ is by definition the equilibrium state of the constant function 0 , whose pressure is $h$. If $h=h_{\mu}$, then $\mu=\mu_{\max }$ and it follows that there is a function $f: S M \rightarrow \mathbb{R}$ with

$$
\log \lambda_{t}^{u}(v)=h t+f\left(g^{t} v\right)-f(v) .
$$

Is it possible to show that $f$ is constant and then that $M$ is locally symmetric? When $M$ is a surface this programme can be carried through using the results of [19].

(5.4) Conjecture (Osserman). Let $0 \geq \kappa_{1}(v) \geq \kappa_{2}(v) \geq \cdots \geq \kappa_{n-1}(v)$ be the eigenvalues of the symmetric map $w \rightarrow R(w, v) v$ on $\{w: w \perp v\}$. Then

$$
h_{\mu} \leq \sum_{i=1}^{n-1}\left[\int_{S M}-\kappa_{i}(v) d \mu\right]^{\frac{1}{2}} \equiv A(M),
$$

with equality if and only if $M$ is locally symmetric.

Conjecture (5.1) would follow if $A(M)$ were also a lower bound for $h$. It is known [25, theorem B] that $h_{\mu} \leq A(M) \leq h$ when $M$ is a surface. Conjecture (5.4) is related to the inequality

$$
\int_{S M} \sum_{i=1}^{n-1}\left(-\kappa_{i}(v)\right)^{\frac{1}{2}} d \mu \leq h_{\mu},
$$

(with equality if and only if $M$ is locally symmetric) proved for negatively curved manifolds by Osserman and Sarnak [34], and extended to rank 1 manifolds independently by Wojtkowski and Ballmann.

(5.5) Green has a conjecture related to (5.1).

(5.5.1) Conjecture [19]. If the mean curvatures of the horospheres through each point of $M$ depend only on the point, then $M$ is locally symmetric.

(5.5.2) It is still unknown whether $M$ must be locally symmetric if all the horospheres have the same mean curvature. 


\section{Problems about rank 1 manifolds}

The idea behind most of these problems is that the fundamental group of a manifold with non-positive curvature and rank 1 should have a strong influence on the geometry and topology of the manifold. Note that if $M$ has rank 1, every metric on $M$ of non-positive curvature also has rank 1 [14].

(6.1) (Eberlein). Let $M$ and $M^{*}$ be compact manifolds with negative curvature. Suppose $\pi_{1}(M) \cong \pi_{1}\left(M^{*}\right)$. Are $M$ and $M^{*}$ homeomorphic? diffeomorphic?

Note that $M$ and $M^{*}$ are homotopy equivalent since their universal covers are contractible; see e.g. [14, pp. 416-417].

(6.1.1) Partial result. Gromov [21] showed that there is a homeomorphism between $S M$ and $S M^{*}$ which conjugates the two geodesic flows.

(6.2) Let $M$ be compact with rank 1. Recall [20], [29] that $\pi_{1}(M)$ contains an abelian subgroup of rank $k$ if and only if $M$ contains a compact $k$-flat. Since all elements of $\pi_{1}(M)$ have infinite order, this implies Preissmann's theorem that if $M$ has negative curvature, then $\pi_{I}(M)$ has the property $(P)$ that every abelian subgroup is isomorphic to $\mathbb{Z}$.

(6.2.1) Question (Eberlein). If $\pi_{1}(M)$ has property (P), does $M$ admit a metric of negative curvature?

$\pi_{1}(M)$ also has property (P) if $M$ satisfies the axiom of uniform visibility introduced in [16], [13]. This follows since $M$ satisfies uniform visibility if and only if it contains no 2 -flat [13, theorem 4.1]. Note that uniform visibility implies rank 1 [11, lemma 2.1]. Heintze and Gromov have constructed rank 1 manifolds whose fundamental groups contain copies of $\mathbb{Z}^{2}[3]$. Here is a weaker form of the previous question.

(6.2.2) Question (Eberlein). If $\pi_{1}(M)$ has property $(P)$, is $M$ a uniform visibility manifold?

This can be restated as

(6.2.2) Question (Eberlein). Can $M$ contain a 2-flat without containing a compact 2-flat?

(6.3) (Eberlein). Suppose $M$ is compact and has rank 1. What does the distribution of free subgroups in $\pi_{1}(M)$ say about the structure of $M$ ?

(6.4) (Ballmann). Suppose $M$ is simply connected and homogeneous. Show that a de Rham factor [28] of $M$ is either symmetric or satisfies uniform visibility.

\section{Structure of the space of metrics of non-positive curvature on rank 1 manifolds} Again $M$ is compact and has rank 1 .

(7.1) Question. Is the space of metrics on $M$ with negative curvature connected?

When $M$ is a surface, there is an easy argument using the fact that the metric is conformally equivalent to one of constant curvature. But what about higher dimensions?

(7.2) Consider the examples of Heintze and Gromov [3]. Their fundamental groups contain copies of $\mathbb{Z}^{2}$, so any metric of non-positive curvature on them must have 
compact 2-flats. Are there any stronger restrictions? V. Schweder showed recently that any metric of non-positive curvature on Gromov's example is locally isometric to a product.

(7.3) Let $(M, \sigma)$ be a locally symmetric manifold with non-constant negative curvature. Then the curvature of $g$ is $1 / 4$-pinched. Suppose $\sigma^{*}$ is another metric on $M$ with 1/4-pinched negative curvature. Is $\left(M, \sigma^{*}\right)$ locally symmetric?

(7.3.1) Recently this question was answered positively by $M$. Ville in the case of a compact or finite volume quotient of $\mathrm{CH}^{2}$, the complex hyperbolic phase.

(7.3.2) Idea of Gromov. Studying the ratios of different characteristic exponents for the geodesic flow of $\sigma^{*}$ should help in comparing the curvature of $\sigma^{*}$ in different directions. The Ruelle inequality relates a global quantity, entropy, to sums of characteristic exponents. Is there a global quantity related to ratios of exponents?

\section{Compact flats in higher rank}

This section is based on $[6, \S 5]$. Let $M$ be compact with rank $\geq 2$. Consider compact regular flats in $M$. These are the analogues of closed geodesics in rank 1. Are there appropriate generalizations of the exponential growth of closed geodesics (4.3.1)? There are several ways to measure the size of a compact flat $F$ : its volume $V(F)$; it systol sys $(F)$, which is the length of the shortest closed geodesic on $F$; and its regular systol rsys $(F)$, which is the length of the shortest regular closed geodesic on $F$. Let:

$$
\begin{aligned}
& N_{V}(t)=\#\{\text { compact regular flats } F: V(F) \leq t\} \\
& N_{S}(t)=\#\{\text { compact regular flats } F: \text { sys }(F) \leq t\} \\
& N_{R S}(t)=\#\{\text { compact regular flats } F: \text { rsys }(F) \leq t\} .
\end{aligned}
$$

There are examples where $N_{S}(t)$ is infinite, but $N_{V}(t)$ and $N_{R S}(t)$ are always finite. (8.1) Question. Do $N_{V}(t)$ and $N_{R S}(t)$ grow exponentially with $t$ ? If so, what are their growth rates?

(8.2) Partial answer. [6, § 5] Let

$$
V R S(t)=\sum_{\operatorname{rsys}(F) \leq t} V(F) .
$$

Then $\operatorname{VRS}(t)$ is always finite and

$$
\liminf _{t \rightarrow \infty} t^{-1} \log \operatorname{VRS}(t) \geq h_{\mu}>0,
$$

where $\mu^{\prime}$ is the restriction of the Liouville measure to the regular vectors.

\section{Structure on the sphere at infinity}

Unless otherwise stated, $H$ is simply connected and $-b^{2} \leq K_{H} \leq-a^{2}<0$.

(9.1) $C^{\alpha}$-structure [2, proposition 2.1]. $H(\infty)$ has a well defined $C^{\alpha}$-structure where $\alpha=a / b$.

(9.1.1) Question (Brin). Does $H(\infty)$ still have a $C^{\alpha}$-structure for some $\alpha \in(0,1)$ if $H$ has rank 1 and $K_{H}$ is bounded below? 
(9.2) Geodesic measures. For $p \in H$, let $\theta_{p}: S_{p} H \rightarrow H(\infty)$ be the map which sends $v$ to the end point of $\gamma_{v}$. Use $\theta_{p}$ to transport Lebesgue measure on $S_{p} H$ to a measure $\mu_{p}$ on $H(\infty)$. We call $\mu_{p}$ a geodesic measure on $H(\infty)$. If $H=\tilde{M}$ for a compact $M$, it follows from the absolute continuity of the stable foliations of the geodesic flow in $S M$ that $\mu_{p}$ and $\mu_{q}$ are equivalent for any $p, q \in H$. The same claim is true if $\boldsymbol{M}$ is compact and has rank 1 .

(9.2.1) Question (Brin). Are all geodesic measures on $H(\infty)$ equivalent if $H=\tilde{M}$ where $M$ has finite volume, rank 1 and curvature bounded from below?

(9.3) Harmonic measures. Consider Brownian motion starting from $p \in H$. Almost every path converges to a point in $H(\infty)$. If $B \subseteq H(\infty)$ is a Borel set, let $P(p, B)$ be the probability that a Brownian path from $p$ converges to a point of $B$. The measures $P(p, \cdot)$ for $p \in H$ are called harmonic measures.

(9.3.1) THEOREM [26], [42]. (i) $P(p, \cdot)$ and $P(q, \cdot)$ are equivalent for any $p, q \in H$.

(ii) For any $B, p \rightarrow P(p, B)$ is a harmonic function on $H$.

(iii) If $\left\{p_{n}\right\} \subseteq H$ converges to $p \in H(\infty)$, the measures $P\left(p_{m}, \cdot\right)$ converge weakly to the probability concentrated at $p$.

(iv) Solution of the Dirichlet problem. Suppose $f: H(\infty) \rightarrow \mathbb{R}$ is continuous. Let

$$
F(p)= \begin{cases}\int_{q \in H(\infty)} f(q) d P(p, q) & \text { if } p \in H, \\ f(p) & \text { if } p \in H(\infty) .\end{cases}
$$

Then $F$ is continuous on $H \cup H(\infty)$ and harmonic on $H$.

The Dirichlet problem is also solved in [1] and [2].

(9.3.2) Question (Brin). What happens to this theory if $H$ has rank 1 and curvature bounded below?

(9.4) Suppose $H=\tilde{M}$ for compact $M$, so the geodesic and harmonic measure classes are well defined. They are obviously equal if $\boldsymbol{M}$ has constant curvature. However, Sullivan [42, p. 724] suggests that they are not equal in general.

Sullivan's question has been answered in dimension two. For, every Riemannian metric $\sigma$ on a surface is conformally equivalent to a metric $\sigma_{0}$ of constant negative curvature and the Laplacian of the metric $\sigma$ is a scalar multiple of the Laplacian for $\sigma_{0}$. Thus, harmonic measures for the two metrics lifted to the universal cover are the same. This reduces the problem to the following question posed by Schoen in connection with the work in [2].

(9.5) Consider the $\operatorname{disc} D \leq\{z \in \mathbb{C}:|z| \leq 1\}$ and $S=\{z \in \mathbb{C}:|z|=1\}$. Let $\sigma$ be a metric on $D$ with $-b^{2} \leq K_{(D, \sigma)} \leq-a^{2}<0$. For $\theta \in S$, let $\phi(\theta)$ be the endpoint of the geodesic ray in $(D, \sigma)$ which is tangent at 0 to the Euclidean ray from 0 to $e^{i \dot{\theta}}$. When is the map $\phi$ singular?

T. Wolff constructed an example of a metric on $D$ with arbitrary sharp pinching for which the map $\phi$ is singular (personal communication). This metric does not allow a compact quotient. Katok proved recently that the map $\phi$ is always singular for a metric of non-constant negative curvature with compact quotient (manuscript in preparation). 
In higher dimension conformal equivalence of metrics does not always take place. Furthermore, it does not guarantee the coincidence of harmonic measures. Thus, both the Sullivan question and the counterpart of (9.5) remain open for manifolds of higher dimension if the metric has compact quotient. However Wolff's construction works in all dimensions.

\section{Time preserving conjugacy of geodesic flows of surfaces}

Let $M_{1}$ and $M_{2}$ be compact surfaces with negative curvature. Let $g_{i}^{i}$ be the geodesic flow on $S M_{i}, i=1,2$, and $h_{i}$ the expanding horocycle flow with the uniform parametrization [32]. Let $\nu_{i}$ be the measure of maximal entropy for $g_{i}^{i}$. Recall that $\nu_{i}$ is the unique ergodic measure for $h_{i}^{t}$ [32].

(10.1) Problem. Suppose there is a $C^{0}$ time-preserving conjugacy $\phi$ between $g_{1}^{t}$ and $g_{2}^{t}$. Show that $M_{1}$ and $M_{2}$ are isometric. For surfaces of constant curvature this follows from [37].

(10.2) Feldman and Ornstein [17] have shown that $\phi$ must be $C^{1}$.

(10.3) The following are equivalent.

(i) There is a $C^{0}$ time-preserving conjugacy $\phi$ between $g_{1}^{t}$ and $g_{2}^{l}$.

(ii) $h_{2}^{t}$ and $h_{2}^{t}$ are measurably conjugate, i.e. there is a measure-preserving map $\psi:\left(S M_{1}, \nu_{1}\right) \rightarrow\left(S M_{2}, \nu_{2}\right)$ such that for $\nu_{1}$-a.e. $v \in S M_{1}$ we have $\psi h_{1}^{t}(v)=h_{2}^{t} \psi(v)$ for all $t \in \mathbb{R}$.

(iii) $M_{1}$ and $M_{2}$ have the same length spectrum; see (3.1).

Proof. (ii) $\Rightarrow$ (i). This is a recent result of Feldman and Ornstein [17].

(iii) $\Rightarrow$ (i). See (3.5).

(i) $\Rightarrow$ (ii). Clearly $\phi$ is a bijection between orbits of $h_{1}^{t}$ and $h_{2}^{t}$. The uniqueness of the uniform parametrization shows that $\phi h_{1}^{t}=h_{2}^{t} \phi$ for all $t$, and unique ergodicity of $h_{i}^{t}$ shows that $\phi$ is measure preserving.

(i) $\Rightarrow$ (iii). Let $\gamma_{i}$ be the element of $\pi_{1}\left(S M_{i}\right)$ determined by one of the fibres of $S M_{i}$. Then $\gamma_{i}$ generates the centre $Z_{i}$ of $\pi_{1}\left(S M_{i}\right)$, and $\pi_{1}\left(M_{i}\right) \cong \pi_{1}\left(S M_{i}\right) / Z_{i}$. Since $\phi_{*}: \pi_{1}\left(S M_{1}\right) \rightarrow \pi_{1}\left(S M_{2}\right)$ is an isomorphism, $\pi_{1}\left(M_{1}\right) \cong \pi_{1}\left(M_{2}\right)$. Since $\phi$ is timepreserving, it is not difficult to show that this isomorphism matches up free homotopy classes containing closed geodesics of the same length. This argument was shown to us by Feldman.

(10.4) Question. What does time-preserving conjugacy of geodesic flows imply in higher dimensions?

\section{General problems}

(11.1) Is there a smooth Riemannian metric on $S^{2}$ with ergodic geodesic flow?

(11.1.1) There is a smooth Finsler metric with ergodic geodesic flow [24]; see also [46].

(11.1.2) R. Osserman (oral communication) constructed an example of a $C^{1}$ metric on $S^{2}$ with ergodic geodesic flow. In that example the curvature is bounded but is discontinuous along several curves. As a next step toward (11.1) it may be interesting to try to construct a metric on $S^{2}$ which is $C^{2}$ everywhere except for finitely many points, and which has ergodic geodesic flow. 
(11.2) (Katok). Are there general mechanisms in Hamiltonian dynamics other than scattering that create ergodicity and positive entropy? For example, are there analogues of the stadium billiard of Bunimovich [10] and more general convex billiards with hyperbolic behaviour recently found by Wojtkowski [45].

\section{REFERENCES}

[1] M. T. Anderson. The Dirichlet problem at infinity for manifolds of negative curvature. J. Diff. Geom. 18 (1983), 701-721.

[2] M. Anderson \& R. Schoen. Positive harmonic functions on complete manifolds of negative curvature. Preprint.

[3] W. Ballman. Axial isometries of manifolds of nonpositive curvature. Math. Ann. 259 (1982), $131-144$.

[4] W. Ballman \& M. Brin. On the ergodicity of geodesic flows. Ergod. Th. and Dynam. Sys. 2 (1982), 311-315.

[5] W. Ballman, M. Brin \& P. Eberlein. Structure of manifolds of non-positive curvature, I. Univ. of Maryland Technical Report TR 84-8 (1984).

[6] W. Ballman, M. Brin \& R. Spatzier. Structure of manifolds of non-positive curvature, II. Univ. of Maryland Technical Report TR84-31 (1984).

[7] M. Berger. Geometry of the spectrum, I. AMS Proc, Sympos. in Pure Math. 27 (1975), Part 2, $129-152$.

[8] R. Bowen. Periodic orbits for hyperbolic flows. Amer. J. Math. 94 (1972), 1-30.

[9] R. Bowen \& D. Ruelle. The ergodic theory of axiom A flows. Inv. Math. 29 (1975), 181-202.

[10] L. A. Bunimovich. On the ergodic properties of nowhere dispersing billiards. Commun. Math. Phys. 65 (1979), 295-312.

[11] K. Burns. Hyperbolic behaviour of geodesic flows on manifolds with no focal points. Ergod. Th. \& Dynam. Sys. 3 (1983), 1-12.

[12] S. S. Chen \& P. Eberlein. Isometry groups of simply connected manifolds of nonpositive curvature. Illinois J. Math. 24 (1980), 73-103.

[13] P. Eberlein. Geodesic flow in certain manifolds without conjugate points. Trans. Amer. Math. Soc. 167 (1972), 151-170.

[14] P. Eberlein. Geodesic rigidity in compact nonpositively curved manifolds. Trans. Amer. Math. Soc. 268 (1981), 411-443.

[15] P. Eberlein. Rigidity of lattices of non-positive curvature. Ergod. Th. \& Dynam. Sys. 3 (1983), 47-85.

[16] P. Eberlein \& B. O’Neill. Visibility manifolds. Pacific J. Math. 46 (1973), 45-109.

[17] J. Feldman \& D. Ornstein. Semi-rigidity of horocycle flows over compact surfaces of variable negative curvature. Preprint (1984).

[18] R. Fricke \& F. Klein. Vorlesungen über die Theorie der Elliptischen Modulfunktionen/Automorphen funktionen. G. Teubner: Leipzig, 1896/1912.

[19] L. W. Green. Remarks on uniformly expanding horocycle parametrizations. J. Diff. Geom. 13 (1978), 263-271.

[20] D. Gromoll \& J. A. Wolf. Some relations between the metric structure and the algebraic structure of the fundamental group in manifolds of nonpositive curvature. Bull. Amer. Math. Soc. 77 (1971), 545-552.

[21] M. Gromov. Three remarks on geodesic dynamics and the fundamental group. Preprint.

[22] V. Guillemin \& D. Kazhdan. Some inverse spectral results for negatively curved 2-manifolds. Topology 19 (1980), 301-312.

[23] S. Hurder. Rigidity of group actions and cocycles. MSRI Berkeley Preprint (1984). To appear in Ergod. Th. \& Dynam. Sys (1985)

[24] A. Katok. Ergodic properties of degenerate integrable Hamiltonian systems. Math. USSR-Izv. 7 (1973), 535-571. Izv. Akad. Nauk. SSSR 37 (1973), 539-576.

[25] A. Katok. Entropy and closed geodesics. Ergod. Th. \& Dynam. Sys. 2 (1982), 339-367.

[26] Yu. I. Kifer. Brownian motion and harmonic functions on manifolds of negative curvature. Th. Probability Appl. 21 (1976), 81-95.

[27] G. Knieper. Das Wachstum der Äquivalenzklassen geschlossener Geodätischer in kompakten Mannigfaltigkeiten. Archiv. Math. 40 (1983), 559-568.

[28] S. Kobayashi \& K. Nomizu. Foundations of Differential Geometry. Vol. 1, Wiley: New York, 1963; 179-193. 
[29] H. B. Lawson \& S. T. Yau. Compact manifolds of non-positive curvature. J. Diff. Geom. 7 (1972), 211-228.

[30] H. P. McKean. Selberg's trace formula as applied to a compact Riemann surface. Comm. Pure Appl. Math. 25 (1972), 225-246.

[31] A. Manning. Topological entropy for geodesic flows. Ann. Math. 110 (1979), 567-573.

[32] B. Marcus. Unique ergodicity of the horocycle flow: variable negative curvature case. Israel J. Math. 21 (1975), $133-144$.

[33] G. A. Margalis. Applications of ergodic theory to the investigation of manifolds of negative curvature. Functional Anal. Appl. 3 (1969), 335-336. Funktsional. Anal. i Prilozhen. 3 (1969), 89-90.

[34] R. Osserman \& P. Sarnak. A new curvature invariant and entropy of geodesic flows. MSRI Berkeley Preprint (1984); Inv. Math. 77 (1984), 455-462.

[35] W. Parry \& M. Pollicott. An analogue of the prime number theorem for closed orbits of axiom A flows. Ann. Math. 118 (1983), 573-591.

[36] Ya. B. Pesin. Geodesic flows with hyperbolic behaviour of the trajectories and objects connected with them. Russian Math. Surveys 36 (1981), 1-59. Uspekhi Mat. Nauk 36 (1981), 3-51.

[37] M. Ratner. Rigidity of horocycle flows. Ann. of Math. 115 (1982), 587-614.

[38] V. Schroeder. Rigidity of locally symmetric spaces in the category of spaces of non-positive curvature. (1983).

[39] Ja. G. Sinai. Gibbs measures in ergodic theory. Russian Math. Surveys 27 (1972), no. 4, $21-69$. Uspekhi Mat. Nauk. SSSR 27 (1972) no. 4, 21-64.

[40] R. J. Spatzier. The geodesic flow and an approach to the classification of manifolds of non-positive curvature. MSRI Berkeley Preprint (1983).

[41] N. Stanton. The heat equation in several complex variables. Bull. Amer. Math. Soc. 11 (1984), 65-84.

[42] D. Sullivan. Dirichlet problem for a negatively curved manifold. J. Diff. Geom. 18 (1983), 723-732.

[43] C. Toll. A multiplicative asymptotic for the prime geodesic theorem. Thesis, Univ. of Maryland. (1984).

[44] M. F. Vignéras. Variétés Riemanniennes isopectrales et non isométriques. Ann. Math. 112 (1980), 21-32.

[45] M. Wojtkowski. Principles for the design of billiards with non-vanishing Lyapunov exponents. Preprint.

[46] W. Ziller. Geometry of the Katok examples. Ergod. Th. \& Dynam. Sys. 3 (1983), 135-157.

[47] W. Ballman. Non-positively curved manifolds of higher rank. University of Maryland Technical Report TR84-38. 